\title{
Role of the rare earth in lattice and magnetic coupling in multiferroic $h-\mathrm{HoMnO}_{3}$
}

\author{
J. Liu, ${ }^{1}$ Y. Gallais, ${ }^{1}$ M.-A. Measson, ${ }^{1}$ A. Sacuto, ${ }^{1}$ S. W. Cheong, ${ }^{2}$ and M. Cazayous ${ }^{1}$ \\ ${ }^{1}$ Laboratoire Matériaux et Phénomènes Quantiques UMR 7162 CNRS, Université Paris Diderot-Paris 7, 75205 Paris cedex 13, France \\ ${ }^{2}$ Rutgers Center for Emergent Materials and Department of Physics and Astronomy, Rutgers University, 136 Frelinghuysen Road, \\ Piscataway, New Jersey 08854, USA
}

(Received 28 February 2017; published 3 May 2017)

\begin{abstract}
We used Raman scattering to study the lattice and magnetic excitations in the hexagonal $\mathrm{HoMnO}_{3}$ single crystals. The $E_{2}$ phonon mode at $237 \mathrm{~cm}^{-1}$ is affected by the magnetic order. This mode is related to the displacement of $\mathrm{Mn}$ and $\mathrm{O}$ ions in the $a-b$ plane and modulates the Mn-O-Mn bond angles in the $a-b$ plane and in the in-plane Mn-Mn superexchange interaction. The mode at $269 \mathrm{~cm}^{-1}$ associated to the displacement of the apical $\mathrm{Ho}^{3+}$ ions along the $c$ direction presents an abrupt change of slope at $T_{N}$, showing that the role of the rare-earth ions cannot be neglected in the magnetic transition. We have identified magnon and crystal-field excitations. The temperature dependence of the magnetic excitations has been compared to the Mn and Ho moment and indicates that the exchange-interaction pattern between $\mathrm{Mn}$ and Ho atoms drives the uniaxial anisotropy gap above the Mn-spin-rotation transition.
\end{abstract}

DOI: 10.1103/PhysRevB.95.195104

\section{INTRODUCTION}

Multiferroics [1], which exhibit coexistence of magnetism and ferroelectricity with cross coupling, are of great scientific and technological interest for further spintronics, magnonics, and nanoelectronic devices. These include new forms of magnetoelectric memories, electrically controlled magnonic elements, or domain-wall-based devices with engineered magnetic structure [2-6]. Additionally, these compounds can combine multiferroic orders and geometrical frustrations at low dimensionality that provide unusual spin dynamics as well as phase transitions [7].

Rare-earth manganites $\left(R \mathrm{MnO}_{3}\right)$ are one of the canonical examples of multiferroics and are one of the most investigated families. These compounds crystallize in two different structures as a function of the ionic radius: orthorhombic structures for larger ionic radius $(R=\mathrm{La}, \mathrm{Ce}, \mathrm{Pr}, \mathrm{Nd}, \mathrm{Sm}, \mathrm{Eu}, \mathrm{Gd}$, $\mathrm{Tb}, \mathrm{Dy}$ ) and hexagonal structure (space group $\mathrm{P}_{3} \mathrm{~cm}$ ) for $R$ with smaller ionic radius $(R=\mathrm{Ho}, \mathrm{Er}, \mathrm{Tm}, \mathrm{Yb}, \mathrm{Lu}, \mathrm{Y})$ [8-10]. Both exhibit multiferrocity, but the microscopic origins are different. The magnetic frustrations in the orthorhombic manganites lead to spin-lattice coupling induced by the inverse Dzyaloshinski-Moriya interaction [9]. The ferroelectricity in the hexagonal compounds results from electrostatic and size effects that lead to the bulking of $\mathrm{MnO}_{5}$ bipyramids and the displacement of the rare-earth ions [11].

For each rare-earth manganite, the interactions between the lattice and magnetic degrees of freedom need to be clarified. Hexagonal $\mathrm{HoMnO}_{3}$ is one of the most studied of the rareearth manganites and has a magnetic field-temperature phase diagram that exhibits a multitude of complex spin structures [12-14]. Recently, the antiferromagnetic spin dynamics in $\mathrm{HoMnO}_{3}$ has been studied, tracking the changes in the magnon modes with Thz pulses [15]. The development of the timeresolved experiments to probe the phonon and spin dynamics show the importance of first understanding the static interactions. Moreover, the stability of a magnetic configuration rather than another and the nature of the spin waves have to be understood. The magnetic properties of $h-\mathrm{HoMnO}_{3}$ rare-earth manganite have been studied by inelastic neutron scattering [16], second harmonic generation [13,14], and low-heat transport [17], whereas the lattice vibrations have been investigated by Raman and infrared [18] spectroscopy. However, the studies of the static coupling between the lattice and magnetic degrees of freedom on $\mathrm{HoMnO}_{3}$ single crystals remain small in number [19,20].

Here, we have investigated the $A_{1}$ and $E_{2}$ phonon modes of $h-\mathrm{HoMnO}_{3}$ with polarized Raman spectroscopy. The temperature dependence of particular phonon modes associated to motions modulating the super-superexchange paths between adjacent $\mathrm{Mn}$ planes and modulating the $\mathrm{Mn}-\mathrm{O}-\mathrm{Ho}$ interactions shows that the $\mathrm{Mn}-\mathrm{Mn}$ and Ho-Mn interaction along the $c$ axis plays an important role in the magnetic ordering in $h-\mathrm{HoMnO}_{3}$. The magnetic excitations have also been measured and spin excitations have been identified. The temperature behavior of the magnon modes shows that the interaction between the rare earth and the $\mathrm{Mn}$ atoms controls the uniaxial anisotropy gap from $T_{N}$ down to the Mn-spin-rotation transition.

\section{EXPERIMENTAL DETAILS}

$\mathrm{HoMnO}_{3}$ single crystals were grown using the hightemperature flux growth technique in a platinum crucible [21]. The crystals are millimeter-size platelets with a thickness around $0.1 \mathrm{~mm}$ and a large surface of $0.5 \mathrm{~cm}^{2}$. The hexagonal $c$ axis is perpendicular to the surface. The crystals have been polished to obtain high surface quality for optical measurements. Raman scattering is performed in backscattering geometry using the $532 \mathrm{~nm}$ excitation line from a laser diode and collected by a triple spectrometer Jobin Yvon T64000 equipped with a liquid-nitrogen-cooled charged-coupled device (CCD). The high rejection rate of the spectrometer allows one to detect the low-frequency excitations. Measurements between 10 and $300 \mathrm{~K}$ have been performed using an ARS closed-cycle $\mathrm{He}$ cryostat.

\section{RESULTS AND DISCUSSION}

$\mathrm{HoMnO}_{3}$ crystallizes in a hexagonal lattice, space group $\mathrm{Pb}_{3} \mathrm{~cm}$. This compound is formed by layers of corner-sharing trigonal $\mathrm{MnO}_{5}$ bipyramids arranged in a layered-type structure 


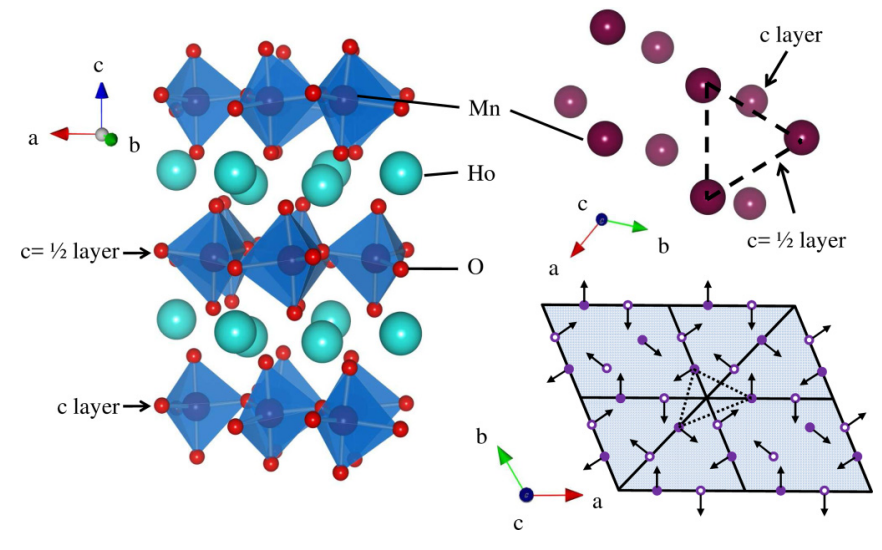

FIG. 1. Crystallographic structure of hexagonal $\mathrm{HoMnO}_{3}$ $\left(\mathrm{P}_{3} \mathrm{~cm}\right.$ phase $)$ and the magnetic order.

in the $a-b$ plane with apical $\left(\mathrm{O}_{1}, \mathrm{O}_{2}\right)$ and in-plane $\left(\mathrm{O}_{3}\right.$, $\mathrm{O}_{4}$ ) oxygen ions as shown in Fig. 1 [22]. Between the bipyramid layers, the rare-earth ion layers are stacked along the $c$ axis. Ferroelectric polarization appears along the $c$ axis below $875 \mathrm{~K}$ and results from electrostatic and size effects that lead to the buckling of $\mathrm{MnO}_{5}$ bipyramids and the displacement of the $\mathrm{Ho}^{3+}$ ions out of the $(a, b)$ plane [11,22]. The moments of $\mathrm{Mn}^{3+}$ ions display antiferromagnetic (AF) orderings at $T_{N, M n}=75 \mathrm{~K}$. The $\mathrm{Mn}^{3+}$ ions form triangular planar sublattices and the AF exchange coupling among $\mathrm{Mn}^{3+}$ moments is geometrically frustrated (see Fig. 1). Below $T_{N, M n}$, the Mn magnetic moments order in $120^{\circ}$ arrangements [23]. Another transition occurs below $40 \mathrm{~K}$ and implies an in-plane Mn spin reorientation by $90^{\circ}[12,13,23]$. Ho moments of the $4 b$ sites are antiferromagnetically coupled within a given layer and $\mathrm{Ho}^{3+}$ ions display AF orderings at $T_{N, H o}=4.6 \mathrm{~K}$. It has been proposed that the $\mathrm{Ho}^{3+}$ moments are oriented along the $c$ axis with an Ising-type anisotropy. The $\mathrm{Ho}^{3+}$ ions present in two different crystallographic sites form two sublattices that can order in parallel or antiparallel orientations $[24,25]$. The $c$ and $c=+1 / 2$ layers are ferromagnetically coupled.

\section{A. Lattice excitations}

The crystal symmetries define the matrix elements of the Raman tensor which are selected by the incident and scattered light polarizations. Thus selection rules define the vibrational modes that can be detected by Raman spectroscopy. The group-theoretical analysis shows that the zone-center phonon modes ( $\Gamma$ point) are in the irreducible representations of the $C_{6 v}: 10 A_{1}+5 A_{2}+10 B_{1}+5 B_{2}+15 E_{1}+15 E_{2}$. Only 38 of these modes are Raman active: $\Gamma_{\text {Raman }}=9 A_{1}+14 E_{1}+$ $15 E_{2}$. Using different scattering configurations, it is possible to choose the mode to activate [26]. Here we used the incident wave vector antiparallel to the scattered one (backscattering configuration). The longitudinal optical $A_{1}$ modes are activated when the phonon propagation direction corresponds to the direction of the ions displacements using the $z(x x)=\bar{z}$ polarization configuration. Pure $E_{2}$ modes are obtained using $z(x y)=\bar{z}$ geometry.

Figure 2 presents the Raman spectra measured on $h-\mathrm{HoMnO}_{3}$ single crystals with the $z(x x)=\bar{z}$ and $z(x y)=\bar{z}$

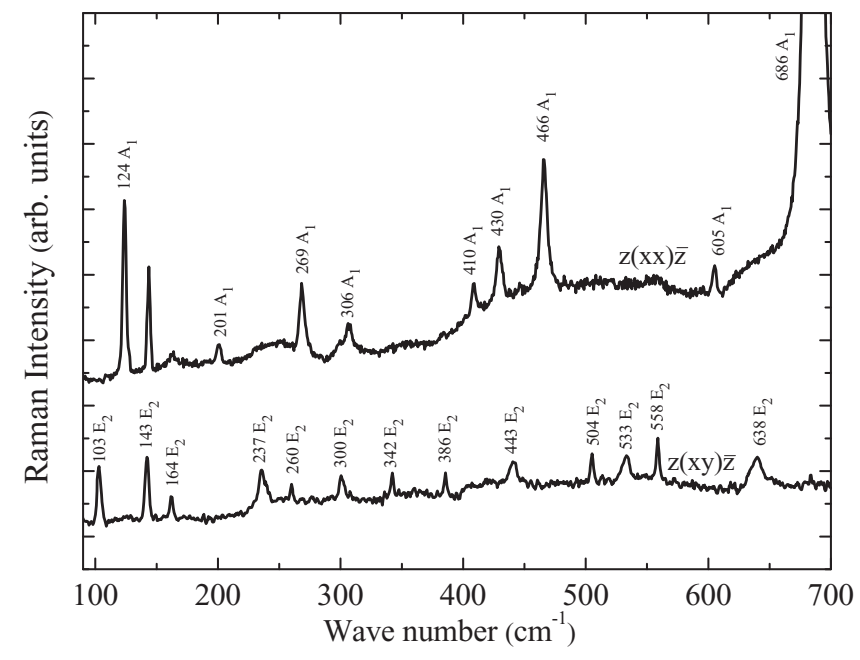

FIG. 2. Phonon modes in $h-\mathrm{HoMnO}_{3}$ single crystal observed at $10 \mathrm{~K}$ using $z(x x)=\bar{z}\left(A_{1}\right.$ modes $)$ and $z(x y)=\bar{z}\left(E_{2}\right.$ modes $)$ scattering configurations.

scattering configurations. The Raman selection rules allow us to identify the $9 A_{1}$ modes and $13 E_{2}$ modes over 15 . The frequencies of the phonon modes at $10 \mathrm{~K}$ are reported in Table I and compared to the previous theoretical results on single crystal [18]. We have measured the temperature dependences of the phonon modes in order to identify the effect of the phase transitions.

TABLE I. $A_{1}$ and $E_{2}$ mode frequencies $\left(\mathrm{cm}^{-1}\right)$ measured in $h-\mathrm{HoMnO}_{3}$ and description of the atomic displacements.

\begin{tabular}{|c|c|c|c|}
\hline Mode & This work & Theory [18] & $\begin{array}{l}\text { Direction of the } \\
\text { largest displacement }\end{array}$ \\
\hline \multirow{9}{*}{$A_{1}$} & 124 & 127 & $z(\mathrm{Ho})$ \\
\hline & 201 & 234 & Rot $x, y\left(\mathrm{MnO}_{5}\right)$ \\
\hline & 269 & 270 & $+z(\mathrm{Ho})-z(\mathrm{Mn})$ \\
\hline & 306 & 295 & $x(\mathrm{Mn}), z\left(\mathrm{O}_{3}\right)$ \\
\hline & 410 & 428 & $+z\left(\mathrm{O}_{3}, \mathrm{O}_{4}\right)+x, y\left(\mathrm{O}_{2}\right)-x, y\left(\mathrm{O}_{1}\right)$ \\
\hline & 430 & 460 & $+z\left(\mathrm{O}_{4}, \mathrm{O}_{3}\right),-z(\mathrm{Mn})$ \\
\hline & 466 & 474 & $+x, y\left(\mathrm{O}_{1}, \mathrm{O}_{2}\right),-x, y(\mathrm{Mn})$ \\
\hline & 605 & 614 & $+z\left(\mathrm{O}_{1}, \mathrm{O}_{2}\right)-z(\mathrm{Mn})$ \\
\hline & 686 & 673 & $+z\left(\mathrm{O}_{1}\right)-z\left(\mathrm{O}_{2}\right)$ \\
\hline \multirow{15}{*}{$E_{2}$} & & 64 & $x, y(\mathrm{Ho}, \mathrm{Mn})$ \\
\hline & 103 & 96 & $+x, y\left(\mathrm{Mn}, \mathrm{O}_{3}, \mathrm{O}_{4}\right)-x, y(\mathrm{Ho})$ \\
\hline & 143 & 137 & $x, y(\mathrm{Ho})$ \\
\hline & 164 & 152 & $x, y(\mathrm{Ho})$ \\
\hline & 237 & 231 & $+x, y(\mathrm{Mn}),-x, y\left(\mathrm{O}_{3}, \mathrm{O}_{4}\right)$ \\
\hline & & 254 & $z\left(\mathrm{Mn}, \mathrm{O}_{2}, \mathrm{O}_{1}\right)$ \\
\hline & 260 & 265 & $z\left(\mathrm{Mn}, \mathrm{O}_{1}, \mathrm{O}_{2}\right)$ \\
\hline & 300 & 330 & $z\left(\mathrm{O}_{2}, \mathrm{O}_{1}\right), x, y\left(\mathrm{O}_{4}\right)$ \\
\hline & 342 & 339 & $+x, y\left(\mathrm{O}_{1}, \mathrm{O}_{2}, \mathrm{O}_{3}, \mathrm{O}_{4}\right)-x, y(\mathrm{Mn})$ \\
\hline & 386 & 402 & $+x, y\left(\mathrm{O}_{1}, \mathrm{O}_{4}\right)-x, y\left(\mathrm{O}_{2}, \mathrm{Mn}\right)$ \\
\hline & 443 & 468 & $+x, y\left(\mathrm{O}_{4}\right)-x, y\left(\mathrm{O}_{1}, \mathrm{Mn}\right)$ \\
\hline & 504 & 523 & $x, y\left(\mathrm{O}_{4}, \mathrm{O}_{3}, \mathrm{O}_{1}, \mathrm{O}_{2}\right)$ \\
\hline & 533 & 557 & $x, y\left(\mathrm{O}_{4}\right)$ \\
\hline & 558 & 583 & $x, y\left(\mathrm{O}_{4}, \mathrm{O}_{3}\right)$ \\
\hline & 638 & 649 & $x, y\left(\mathrm{O}_{3}, \mathrm{O}_{4}\right)$ \\
\hline
\end{tabular}



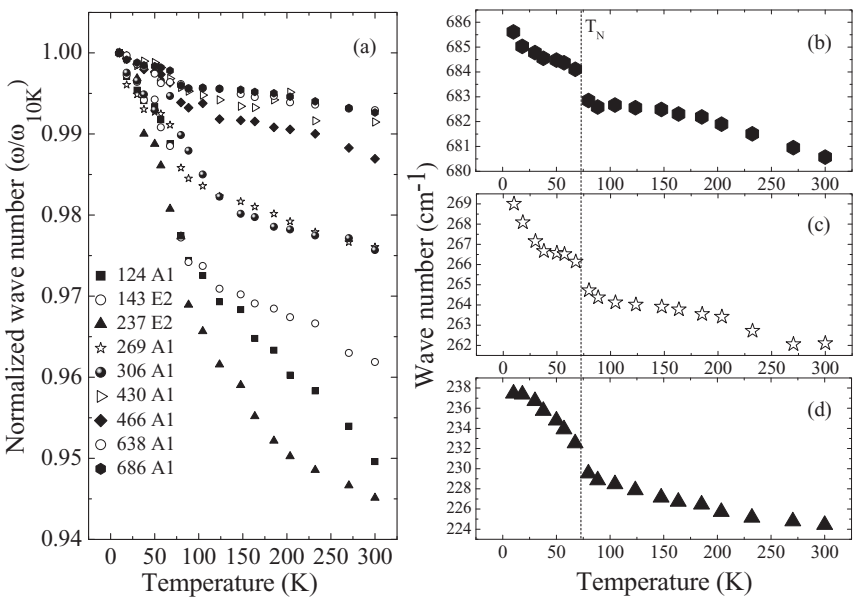

FIG. 3. (a) Normalized wave numbers $[\omega(\mathrm{T}) / \omega(10 \mathrm{~K})]$ of $7 A_{1}$ and $2 E_{2}$ modes and wave numbers of the (b) $A_{1}$ mode at $686 \mathrm{~cm}^{-1}$, (c) $A_{1}$ mode at $269 \mathrm{~cm}^{-1}$, and (d) $E_{2}$ mode at $237 \mathrm{~cm}^{-1}$ as a function of temperature.

Figure 3(a) presents the normalized frequencies over the frequency at $10 \mathrm{~K}$ of several $A_{1}$ and $E_{2}$ modes as a function of temperature. All the phonon frequencies soften due to the dilation of the unit cell when temperature increases. Three phonon modes present an abrupt change in their frequencies, as shown in Figs. 3(b)-3(d) as the fingerprint of the spin-phonon coupling in the magnetically ordered phase.

The $E_{2}$ mode at $237 \mathrm{~cm}^{-1}$ presents a frequency shift beyond the mean behavior of the other modes [Fig. 3(a)] and an abrupt change of slope around the Néel temperature in Fig. 3(d). This phonon mode is related to the displacement of $\mathrm{Mn}$ and $\mathrm{O}$ ions in the $a-b$ plane and it modulates the in-plane $\mathrm{Mn}-\mathrm{Mn}$ superexchange interaction and the $\mathrm{Mn}-\mathrm{O}-\mathrm{Mn}$ bond angles in the $a-b$ plane. Such a behavior has already been measured in rare-earth manganites, such as in $\mathrm{YbMnO}_{3}$ single crystals [27]. The peaks at 269 and $686 \mathrm{~cm}^{-1}$ show the same behavior at the Néel temperature.

The mode at $269 \mathrm{~cm}^{-1}$ is associated to the relative displacement of the apical $\mathrm{Ho}^{3+}$ ions along the $c$ direction. The role played by the $R$ element in the $R \mathrm{MnO}_{3}$ manganites has been underestimated. However, the role of the nonmagnetic $R$ atom is not negligible as underlined by the temperature behavior of the $\mathrm{Ho}^{3+}$ ions frequency at the the Néel temperature.

The mode at $686 \mathrm{~cm}^{-1}$ is related to the relative displacement of the apical oxygen ions along the $c$ direction and modulates the Mn-O-O-Mn bond angles. Notice that the interplanar coupling between $\mathrm{Mn}$ cations and two $\mathrm{O}$ anions (Mn-O-O-M) corresponds to the interplanar super-superexchange paths. The abrupt change of slope at $T_{N}$ shows that the supersuperexchange path through the apical oxygen participates in the three-dimensional magnetic ordering.

\section{B. Spin excitations}

Figure 4(b) shows the low-frequency Raman spectra measured on a $h-\mathrm{HoMnO}_{3}$ single crystal at $10 \mathrm{~K}$. Three peaks are measured: $P_{1}=13 \mathrm{~cm}^{-1}, P_{3}=25 \mathrm{~cm}^{-1}, P_{2}=41 \mathrm{~cm}^{-1}$ with two polarization configurations. Remember that Raman scattering can probe, in addition to phonon modes, the crystal
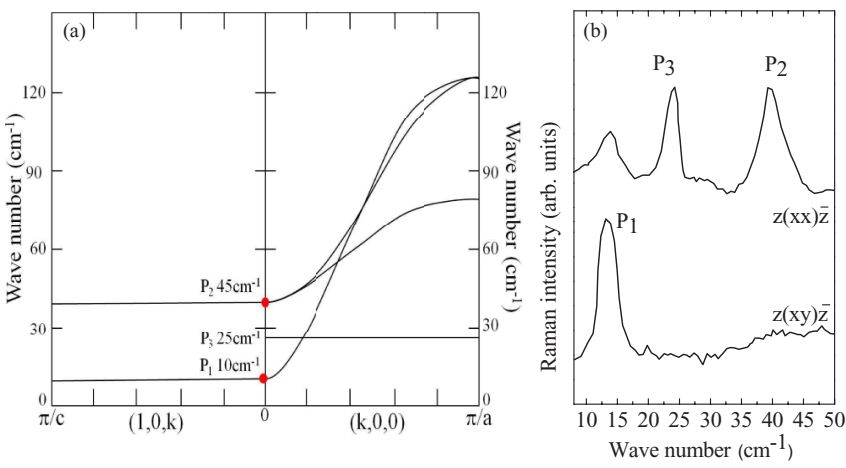

FIG. 4. (a) Magnon dispersions along $\mathbf{k}=(1,0, k)$ and $\mathbf{k}=(k, 0,0)$ extracted from Ref. [16]. (b) Raman spectra of low-frequency excitations measured at $10 \mathrm{~K}$ in $z(x x) \bar{z}$ and $z(x y) \bar{z}$ configuration.

field, one magnon (zone-center) excitations, and two-magnon (zone-edge) excitations. Below $50 \mathrm{~cm}^{-1}$, there are no phonon modes and, as shown in the following paragraph, the $P_{1}, P_{2}$, and $P_{3}$ peaks disappear at the Néel temperature, so these peaks can be thus attributed to magnetic excitations. In order to determine the origin of the magnetic excitations, we have compared our measurements to neutron-scattering results. Figure 4(a) represents the magnetic excitation dispersions along the $\mathbf{k}=(1,0, k)$ and $\mathbf{k}=(k, 0,0)$ axes [16]. At the $\Gamma$ point, the mode at $10 \mathrm{~cm}^{-1}$ corresponds to the uniaxial anisotropy gap and can be associated to the $P_{1}$ peak. The mode at $45 \mathrm{~cm}^{-1}$ in Fig. 4(a) is split at higher energies at the zone edge with a higher branch around $130 \mathrm{~cm}^{-1}$ and a lower branch around $80 \mathrm{~cm}^{-1}$. The $P_{2}$ peak can be attributed to this mode and corresponds to the one-magnon mode of the $\mathrm{Mn}^{3+}$ magnetic structure in the $(a, b)$ plane, in particular to the global in-phase and out-of-phase rotations of the $120^{\circ}$ pattern inside the basal plane. The $P_{3}$ is related to the crystal-field excitation at $25 \mathrm{~cm}^{-1}$, which presents a flat dispersion as shown by neutron scattering [16]. Transition from the ground state of
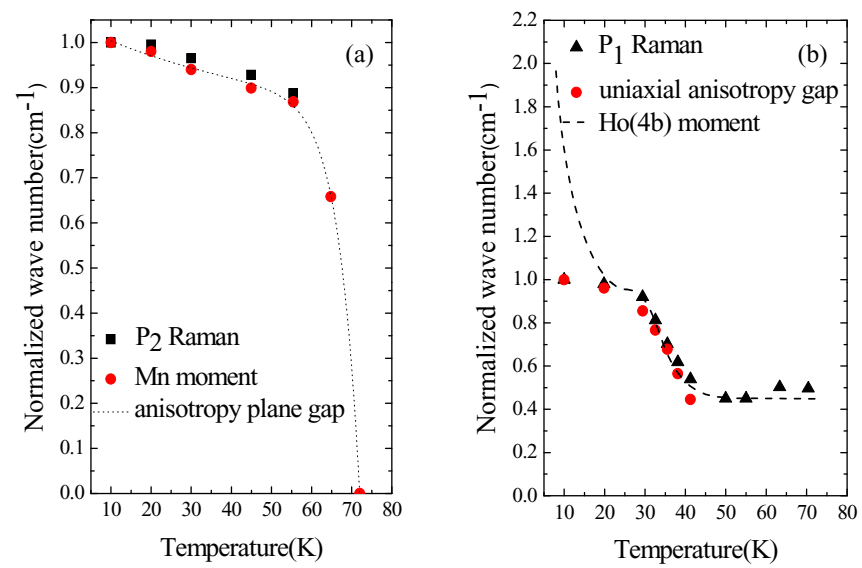

FIG. 5. Temperature dependence of normalized wave number $[\omega(\mathrm{T}) / \omega(10 \mathrm{~K})]$ of (a) the magnetic mode $P_{2}$ compared with the Mn moments and the energy of the anisotropy plane gap [28], and of (b) the $P_{1}$ mode compared to the uniaxial energy gap and the $\mathrm{Ho}(4 b)$ moment [28]. 
the $4 f$ ions to the excited state are, in principle, Raman active and correspond to crystal-field excitations.

The temperature dependence of the magnon wave number $P_{1}$ and $P_{2}$ recorded from 10 to $70 \mathrm{~K}$ is shown in Fig. 5 .

In Fig. 5(a), the Raman shift of the $P_{2}$ mode decreases as the temperature is increased. This conventional softening is first compared to the one of the Mn moment. The good agreement with the variation of the Mn moment shows that this magnon mode can be thus associated to the spin excitations of the $\mathrm{Mn}^{3+}$ ions. It also agrees well with the temperature dependence of the anisotropy plane gap measured by neutron scattering [28]. The $P_{2}$ peak is the signature of the anisotropy planar gap in the $(a, b)$ plane and varies with temperature similar to the $\mathrm{Mn}^{3+}$ ion moment.

In Fig. 5(b), the $P_{1}$ peak wave number presents a change in the slope between 30 and $40 \mathrm{~K}$. We have compared this behavior to the thermal evolution of the uniaxial gap [28]. The good agreement confirms that this peak is related to the uniaxial anisotropy gap. At $T_{S R}=33 \mathrm{~K}$, a Mn-spin-rotation transition has been observed, implying an in-plane Mn-spin reorientation by $90^{\circ}$ and an antiferromagnetic order of the Ho moments along the $c$ axis. To shed some light on the role of the Ho ions, we have drawn in Fig. 5(b) the temperature dependence of Ho moments. The $P_{1}$ peak frequency shift is close to the behavior of the Ho moment down to $T_{S R}$. This evidence points out the role of the $\mathrm{Ho}^{3+}$ ion moment and of the Ho-Mn interaction on the $P_{1}$ spin wave. Below $T_{S R}$, the uniaxial gap is no longer correlated to the Ho moment. Those measurements show that the exchange-interaction pattern between the rare earth and $\mathrm{Mn}$ atoms is a key to understand the uniaxial anisotropy gap but not sufficient below the Mn-spin-rotation transition.

\section{CONCLUSION}

In summary, our measurements show that the $E_{2}$ mode associated to the relative displacement of the apical $\mathrm{Ho}^{3+}$ ions along the $c$ direction is sensitive to the the Néel temperature, pointing out the non-negligible role played by the $R$ element in the $R \mathrm{MnO}_{3}$ manganites. We also reveal the spin excitations and the role played by the $\mathrm{Ho}^{3+}$ ion moment and of the Ho-Mn interaction on the uniaxial anisotropy gap.
[1] W. Eerenstein, N. D. Mathur, and J. F. Scott, Nature (London) 442, 759 (2006).

[2] J. Allibe, S. Fusil, K. Bouzehouane, C. Daumont, D. Sando, E. Jacquet, C. Deranlot, M. Bibes, and A. Barthélémy, Nano Lett. 12, 1141 (2012).

[3] P. Rovillain, R. De Sousa, Y. Gallais, A. Sacuto, M. Méasson, D. Colson, A. Forget, M. Bibes, A. Barthélémy, and M. Cazayous, Nat. Mater. 9, 975 (2010).

[4] Y. Kajiwara, K. Harii, S. Takahashi, J. Ohe1, K. Uchida, M. Mizuguchi, H. Umezawa, H. Kawai, K. Ando, K. Takanashi, S. Maekawa, and E. Saitoh, Nature (London) 464, 262 (2010).

[5] S. O. Demokritov and A. N. Slavin, Magnonics: From Fundamentals to Applications (Springer-Verlag, Berlin, 2013).

[6] J. H. Lee, I. Fina, X. Marti, Y. H. Kim, D. Hesse, and M. Alexe, Adv. Mater. 26, 7078 (2014).

[7] T. Kimura, T. Goto, H. Shintani, K. Ishizaka, T. Arima, and Y. Tokura, Nature (London) 426, 55 (2003).

[8] M. Fiebig, J. Phys. D 38, R123 (2005).

[9] S. W. Cheong and M. Mostovoy, Nat. Mater. 6, 13 (2007).

[10] J. Park, J. G. Park, G. S. Jeon, H. Y. Choi, C. H. Lee, W. Jo, R. Bewley, K. A. McEwen, and T. G. Perring, Phys. Rev. B 68, 104426 (2003).

[11] B. B. Van Aken, T. T. M. Palstra, A. Filippetti, and N. A. Spaldin, Nat. Mater. 3, 164 (2004).

[12] A. Muñoz, J. A. Alonso, M. J. Martinez-Lope, M. T. Casais, J. L. Martinez, and M. T. Fernandez-Diaz, Chem. Mater. 13, 1497 (2001).

[13] M. Fiebig, D. Fröhlich, K. Kohn, S. Leute, Th. Lottermoser, V. V. Pavlov, and R. V. Pisarev, Phys. Rev. Lett. 84, 5620 (2000).

[14] M. Fiebig, C. Degenhardt, and R. V. Pisarev, J. Appl. Phys. 91, 8867 (2002).
[15] P. Bowlan, S. A. Trugman, J. Bowlan, J. X. Zhu, N. J. Hur, A. J. Taylor, D. A. Yarotski, and R. P. Prasankumar, Phys. Rev. B 94, 100404 (2016).

[16] X. Fabrèges, S. Petit, I. Mirebeau, S. Pailhes, L. Pinsard, A. Forget, M. T. Fernandez-Diaz, and F. Porcher, Phys. Rev. Lett. 103, 067204 (2009).

[17] X. M. Wang, C. Fan, Z. Y. Zhao, W. Tao, X. G. Liu, W. P. Ke, X. Zhao, and X. F. Sun, Phys. Rev. B 82, 094405 (2010).

[18] A. P. Litvinchuk, M. N. Iliev, V. N. Popov, and M. M. Gospodinov, J. Phys.: Condens. Matter 16, 809 (2004).

[19] C. dela Cruz, F. Yen, B. Lorenz, Y. Q. Wang, Y. Y. Sun, M. M. M. Gospodinov, and C. W. Chu, Phys. Rev. B 71, 060407(R) (2005).

[20] M. Poirier, J. C. Lemyre, P. O. Lahaie, L. Pinsard-Gaudart, and A. Revcolevschi, Phys. Rev. B 83, 054418 (2011).

[21] T. Choi, Y. Horibe, H. T. Yi, Y. J. Choi, W. Wu and S.-W. Cheong, Nat. Mater. 9, 423 (2010).

[22] T. Lottermoser, T. Lonkai, U. Amann, D. Hohlwein, J. Ihringer, and M. Fiebig, Nature (London) 430, 541 (2004).

[23] O. P. Vajk, M. Kenzelmann, J. W. Lynn, S. B. Kim, and S.-W. Cheong, Phys. Rev. Lett. 94, 087601 (2005).

[24] P. J. Brown and T. Chatterji, Phys. Rev. B 77, 104407 (2008).

[25] N. Hur, I. K. Jeong, M. F. Hundley, S. B. Kim, and S. W. Cheong, Phys. Rev. B 79, 134120 (2009).

[26] S. P. S. Porto, J. A. Giordmaine, and T. C. Damen, Phys. Rev. 147, 608 (1966).

[27] J. Liu, C. Toulouse, P. Rovillain, M. Cazayous, Y. Gallais, M.-A. Measson, N. Lee, S. W. Cheong, and A. Sacuto, Phys. Rev. B 86, 184410 (2012)

[28] X. Fabrèges, Ph.D. thesis, University Paris-Sud 11, France, 2010. 